\section{Zahlen des Monats}

\section{Schlecht informiert}

Nur sieben Prozent der Deutschen wissen, welches Organ die Leistungsansprüche für Medikamente der gesetzlich Versicherten, die nicht bereits gesetzlich geregelt sind, festlegt. Das hat eine Studie zur Transparenz von Kassenleistungen ergeben. Der Gemeinsame Bundesausschuss und seine Arbeit, ebenso der Leistungskatalog der gesetzlichen Krankenversicherung sind demnach vielen Deutschen weitgehend unbekannt. 76 Prozent der Befragten wünschen sich mehr Mitbestimmung im Gesundheitssystem.

\section{Gut gefüllt}

51,627 Millionen Zahnfüllungen wurden im Jahr 2015 in Deutschland über die Kassenzahnärztlichen Vereinigungen mit der gesetzlichen Krankenversicherung abgerechnet. Dies geht aus dem Jahrbuch 2016 der Kassenzahnärztlichen Bundesvereinigung hervor. Damit setzt sich der tendenzielle Rückgang der Anzahl der Füllungen um 2,2 Prozent je Mitglied gegenüber dem Vorjahr fort.

\section{Süße Hauptstadt}

Die Bundesbürger leben anscheinend durchaus gesundheitsbewusst, wie eine Umfrage im Auftrag der Bundesvereinigung Deutscher Apothekerverbände nahelegt: Demnach treibt mehr als jeder vierte Deutsche täglich oder fast täglich mindestens eine halbe Stunde Sport. Allerdings liegt die Raucherquote bei 28 Prozent. Die meisten Raucher leben in NRW, in Hessen rauchen die wenigsten. Die Berliner essen am wenigsten Fleisch, dafür am häufigsten Süßigkeiten.

mar

Allianz für Gesundheitskompetenz

\title{
14 Partner zur Stärkung des Gesundheitswissens
}

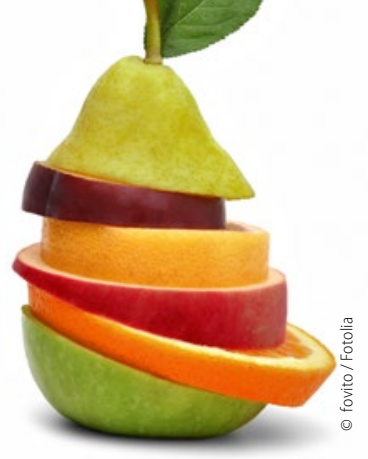

Mit dem Wissen um die eigene Gesundheit steht es in Deutschland offenbar nicht zum Besten. Deshalb hat das Bundesgesundheitsministerium (BMG) mit 14 Organisationen, politischen Institutionen und Körperschaften aus dem Gesundheitswesen eine „Allianz für Gesundheitskompetenz" geschlossen. Im Mittelpunkt der Allianz soll die Kommunikation zwischen Ärzten und Patienten stehen. Bundesgesundheitsminister Hermann Gröhe mahnte dringend „mehr verständliche Gesundheitsinformationen“ an. „Es ist nicht immer einfach, im Dickicht der oft unverständlichen Gesundheitsinformationen den Durchblick zu bewahren." Ein gemeinsamer Kraftakt sei nötig. Laut einer aktuellen Studie verfügten mehr als die Hälfte der Menschen in Deutschland (rund 54 Prozent) nur über eine „eingeschränkte Gesundheitskompetenz", teilte das BMG mit. Dies wirke sich nicht nur auf richtige Entscheidungen zu gesunder Lebensweise und Krankheitsbewältigung aus, sondern auch wirtschaftlich. Nach Schätzungen der Weltgesundheitsorganisation WHO werden drei bis fünf Prozent der Gesundheitsausgaben durch unzureichende Gesundheitskompetenz verursacht. Für Deutschland bedeutet dies laut BMG neun bis 15 Milliarden Euro.

Auch die Kassenzahnärztliche Bundesvereinigung und die Bundeszahnärzte- kammer haben die gemeinsame Erklärung zur Allianz für Gesundheitskompetenz mit unterzeichnet. Die Aktivitäten dienten einer Gesamtstrategie der Vertragszahnärzteschaft zur Verbesserung der Mundgesundheitskompetenz, hieß es von der KZBV, die ihre Aktivitäten nach eigenen Angaben in diesem Bereich neu bündelt und vertieft. Die BZÄK verwies auf die aktive Mitarbeit der Patienten. Diese könne allerdings nur durch umfassende Aufklärung und Information gelingen.

Der Bundesvorsitzende des Freien Verbandes Deutscher Zahnärzte (FVDZ), Harald Schrader, bewertet die Allianz positiv, allerdings müsse sie noch mit Leben gefüllt werden. „Die Initiative zeigt aber auch, wie wichtig es ist, die Verantwortung für eine Therapieentscheidung wieder weitgehend in die Praxis zu verlegen und dem Patienten mehr Eigenverantwortung für seine eigene Mundgesundheit zuzutrauen“, sagte Schrader. Das vom FVDZ entwickelte Modell zur Patientensouveränität könne gerade im Bereich der Zahnheilkunde beispielhaft angewendet werden, denn es setze auf Information, individuelle Beratung und individuelle Prophylaxe- oder Behandlungslösungen. „Mehr gesundheitliche Kompetenz macht unsere Patienten zu eigenverantwortlichen Patienten“, betont Schrader.

sas

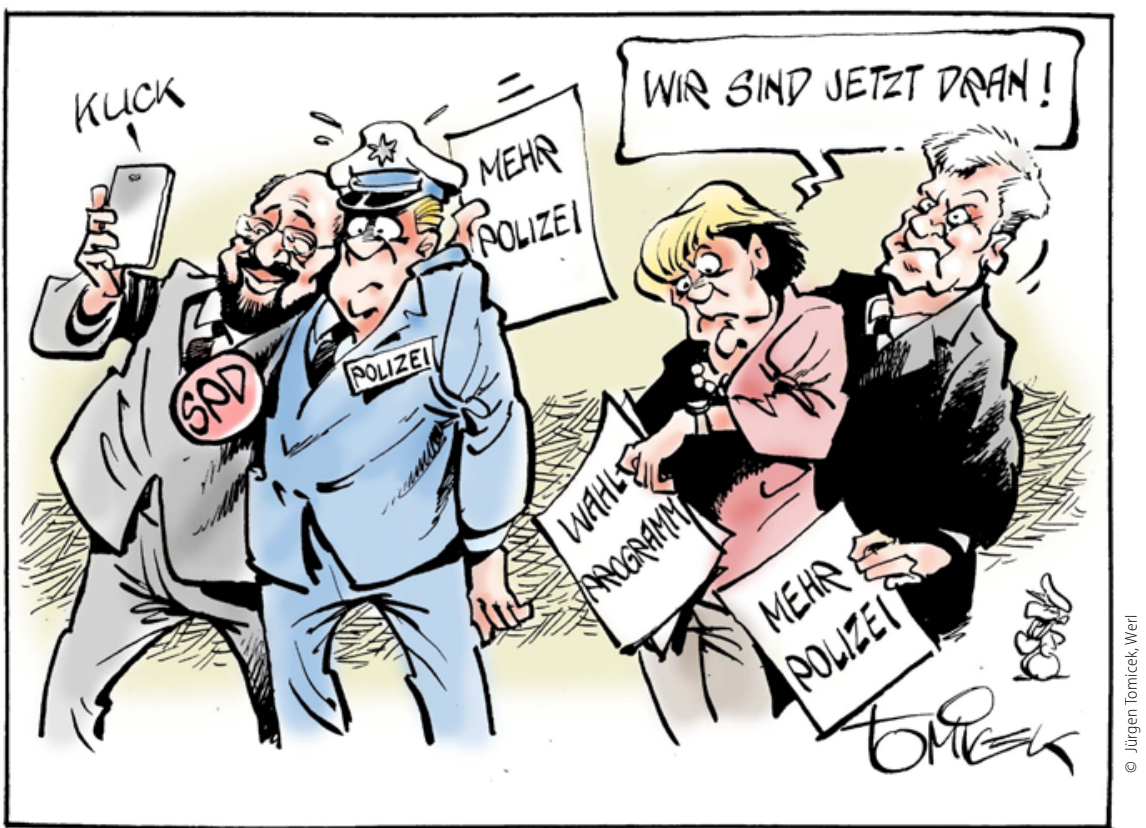

